Article

\title{
Annexin A1 Expression Is Associated with Epithelial-Mesenchymal Transition (EMT), Cell Proliferation, Prognosis, and Drug Response in Pancreatic Cancer
}

\author{
Masanori Oshi 1,2®) Yoshihisa Tokumaru 1,3®D, Swagoto Mukhopadhyay ${ }^{1}$, Li Yan ${ }^{4}$, Ryusei Matsuyama ${ }^{2}$, \\ Itaru Endo ${ }^{2}$ and Kazuaki Takabe ${ }^{1,2,5,6,7,8, *(1)}$
}

check for updates

Citation: Oshi, M.; Tokumaru, Y.; Mukhopadhyay, S.; Yan, L.; Matsuyama, R.; Endo, I.; Takabe, K. Annexin A1 Expression Is Associated with Epithelial-Mesenchymal Transition (EMT), Cell Proliferation, Prognosis, and Drug Response in Pancreatic Cancer. Cells 2021, 10, 653. https://doi.org/10.3390/cells1003 0653

Academic Editor: Antonello Petrella

Received: 2 February 2021

Accepted: 12 March 2021

Published: 15 March 2021

Publisher's Note: MDPI stays neutral with regard to jurisdictional claims in published maps and institutional affiliations.

Copyright: (c) 2021 by the authors. Licensee MDPI, Basel, Switzerland. This article is an open access article distributed under the terms and conditions of the Creative Commons Attribution (CC BY) license (https:/ / creativecommons.org/licenses/by/ $4.0 /)$.
1 Department of Surgical Oncology, Roswell Park Comprehensive Cancer Center, Buffalo, NY 14263, USA; masa1101oshi@gmail.com (M.O.); Yoshihisa.Tokumaru@roswellpark.org (Y.T.); Swagoto.Mukhopadhyay@RoswellPark.org (S.M.)

2 Department of Gastroenterological Surgery, Yokohama City University School of Medicine, Yokohama, Kanagawa 236-0004, Japan; ryusei@yokohama-cu.ac.jp (R.M.); endoit@med.yokohama-cu.ac.jp (I.E.)

3 Department of Surgical Oncology, Graduate School of Medicine, Gifu University, 1-1 Yanagido, Gifu 501-1194, Japan

4 Department of Biostatistics \& Bioinformatics, Roswell Park Comprehensive Cancer Center, Buffalo, NY 14263, USA; li.yan@roswellpark.org

5 Department of Gastrointestinal Tract Surgery, Fukushima Medical University School of Medicine, Fukushima 960-1295, Japan

6 Department of Surgery, Jacobs School of Medicine and Biomedical Sciences, University at Buffalo the State University of New York, Buffalo, NY 14263, USA

7 Department of Surgery, Niigata University Graduate School of Medical and Dental Sciences, Niigata 951-8510, Japan

8 Department of Breast Surgery and Oncology, Tokyo Medical University, Tokyo 160-8402, Japan

* Correspondence: kazuaki.takabe@roswellpark.org; Tel.: +1-716-8-455-540; Fax: +1-716-8-451-668

\begin{abstract}
Annexin A1 (ANXA1) is a calcium-dependent phospholipid-binding protein overexpressed in pancreatic cancer (PC). ANXA1 expression has been shown to take part in a wide variety of cancer biology, including carcinogenesis, cell proliferation, invasion, apoptosis, and metastasis, in addition to the initially identified anti-inflammatory effect in experimental settings. We hypothesized that ANXA1 expression is associated with cell proliferation and survival in PC patients. To test this hypothesis, we analyzed 239 PC patients in The Cancer Genome Atlas (TCGA) and GSE57495 cohorts. ANXA1 expression correlated with epithelial-mesenchymal transition (EMT) but weakly with angiogenesis in PC patients. ANXA1-high PC was significantly associated with a high fraction of fibroblasts and keratinocytes in the tumor microenvironment. ANXA1 high PC enriched multiple malignant gene sets, including hypoxia, tumor necrosis factor (TNF)- $\alpha$ signaling via nuclear factorkappa B (NF-kB), and MTORC1, as well as apoptosis, protein secretion, glycolysis, and the androgen response gene sets consistently in both cohorts. ANXA1 expression was associated with TP53 mutation alone but associated with all KRAS, p53, E2F, and transforming growth factor (TGF)- $\beta$ signaling pathways and also associated with homologous recombination deficiency in the TCGA cohort. ANXA1 high PC was associated with a high infiltration of T-helper type 2 cells in the TME, with advanced histological grade and MKI67 expression, as well as with a worse prognosis regardless of the grade. ANXA1 expression correlated with a sensitivity to gemcitabine, doxorubicin, and 5-fluorouracil in PC cell lines. In conclusion, ANXA1 expression is associated with EMT, cell proliferation, survival, and the drug response in $\mathrm{PC}$.
\end{abstract}

Keywords: ANXA1; biomarker; gene set; GSEA; metastasis; pancreatic cancer; survival; treatment response; tumor microenvironment 


\section{Introduction}

Pancreatic cancer (PC) ranks as the fourth most common cause of cancer-related deaths worldwide. Its five-year overall survival is less than $5 \%$ and is regarded as one of the most devastating cancer diagnoses [1]. Numerous studies over the past decades have targeted PC biology and uncovered mutations in KRAS, p53, cyclin-dependent kinase inhibitor 2A $(C D K N 2 A)$, and SMAD4 and their signaling pathways. These signaling pathways and the associated aberrant activation of genes play critical roles in PC progression [2]. However, the clinical relevance of these basic science findings remains vague due to a lack of studies using large patient cohorts. Recent advances in the high-volume comprehensive genomic sequencing of human tumor samples can help link the PC underlying mechanisms with clinical practice. Analyses using algorithms on comprehensive transcriptomes enable a deeper understanding of the clinical relevance of various signaling pathways and immune status within human cancers. For example, the Gene Set Variation Analysis (GSVA) allows us to understand multiple signaling pathways' biological activity [3]. The xCell algorithm permits us to measure the fractions of 64 infiltrating cell types in the tumor microenvironment (TME) [4]. This approach has already yielded several candidates for prognostic biomarkers. Yamazaki et al. reported that epithelial-mesenchymal transition (EMT) activity in PC is a promising prognostic biomarker. Our group reported that high activity of the G2M checkpoint pathway [5] and lympho-vascular invasion [6] is associated with worse survival. In contrast, the abundance of mature blood vessels [7] and fibroblasts in PC [8] is associated with better survival. The transcriptome analysis may also uncover potential therapeutic targets for PC.

Annexin A1 (ANXA1, also known as lipocortin I) is a member of the annexin family of calcium-dependent phospholipid-binding proteins located on the cytosolic face of the plasma membrane and inhibits phospholipase A2 [9,10]. ANXA1 preserves the cytoskeleton integrity and plays a significant role in the malignant phenotypes of cancer cells in vitro [11]. ANXA1 is known to play a wide variety of functions in cancer biology, including carcinogenesis, cell proliferation, apoptosis, invasion, and metastasis, in addition to an anti-inflammatory effect [12,13]. ANXA1 regulates transforming growth factor (TGF)$\beta$ signaling and promotes epithelial-mesenchymal transition (EMT) [14]. We previously reported that the high expression of ANXA1 is significantly associated with inflammation, angiogenesis, and mast cell infiltration in breast cancer using in silico analyses [15]. Some suggest ANXA1 is an attractive prognostic and predictive marker of PC due to its role in metastasis based upon in vivo experiments [11]. In addition to its relationship with cancer cells, ANXA1 expression is also associated with multiple cells in the TME, such as fibroblasts, and, with angiogenesis, the generation of new vessels and metastasis [16,17]. Novizio et al. reported that the ANXA1 extracellular vesicle (EV) complex participates in tumor cells-stroma intercommunication as a vehicle during PC progression, suggesting that ANXA1 may have potential prognostic and diagnostic roles [18].

Here, we hypothesized that ANXA1 expression is associated with cell proliferation and survival in PC and tested this hypothesis using multiple large patient cohorts.

\section{Results}

2.1. Annexin A1 (ANXA1) Expression Correlates with Epithelial-Mesenchymal Transition (EMT) but Not with Angiogenesis or Mature Vessel Formation in Pancreatic Cancer (PC)

Since ANXA1 expression was linked to EMT in multiple cancer types [19-21], we first investigated the relationship between ANXA1 expression and EMT in PC. The EMT pathway activity was measured using the gene set variation analysis (GSVA) algorithm, following the method we previously reported [5,22-24]. Concordantly, we found that ANXA1 expression significantly correlated with the EMT pathway score in PC consistently in both The Cancer Genome Atlas (TCGA) and GSE57495 cohorts (Figure 1A; Spearman's rank correlations $(r)=0.453$ and 0.536 , respectively; all $p<0.01$ ). The low and high expression of ANXA1 was determined by the median within each cohort (Figure S1). Further, EMT-associated genes, CDH1 (Cadherin 1), CDH2, SNAI1 (Snail Family Transcriptional 
Repressor 1), SNAI2, and TWIST1 (twist family BHLH transcription factor 1) were all elevated in ANXA1 high PC consistently in both cohorts, except for CDH1 in the GSE57495 cohort. We found that other EMT-associated genes, including FN1 (Fibronectin 1), VIM (Vimentin), and TGFBI (transforming growth factor, beta-induced), were also significantly elevated in ANXA1 high PC in both cohorts (Figure S2). Further, we found that almost all of the expressions of genes that constitute the EMT pathway were significantly correlated with ANXA1 expression (Table S1). We previously published that ANXA1 expression was associated with angiogenesis in breast cancer [15] and that the abundance of mature blood vessels was associated with better survival [7]; thus, it was of interest whether this was the case in PC. We found that ANXA1 expression was weakly correlated with the angiogenesis score measured by the GSVA algorithm consistently in both the TCGA and GSE57495 cohorts (Figure 1C). There was no association between the ANXA1 expression and angiogenesis-related cells such as endothelial cells, microvascular (mv), and lymphatic (ly) endothelial cells, except for mv endothelial cells in the GSE57495 cohort (Figure 1D). Generally, there was no association between ANXA1 expression and mature blood vesselrelated gene expressions, such as PECAM1 and S1PR1, except for PECAM1 in the TCGA cohort (Figure 1E). However, ANXA1 high PC was significantly associated with a high fraction of fibroblasts and keratinocytes in both cohorts (Figure 1F). These results show that ANXA1 expression is associated with EMT but only weakly with angiogenesis.

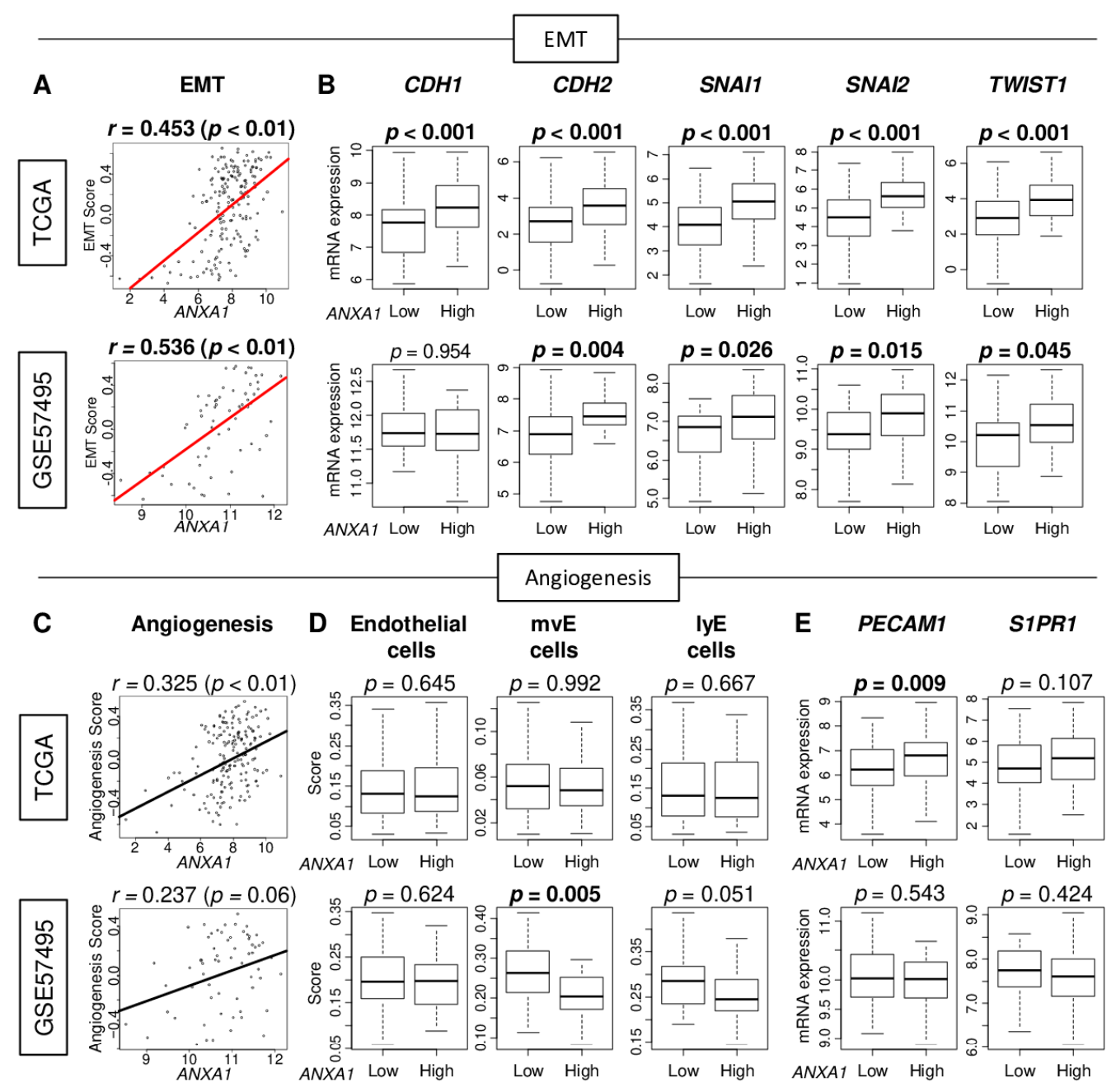

Figure 1. Cont. 


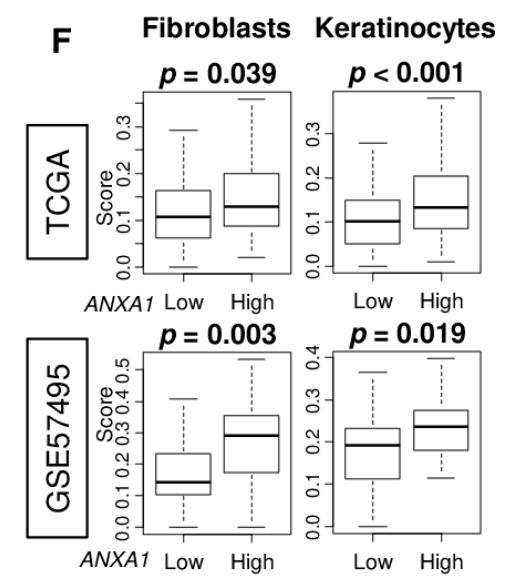

Figure 1. Association of Annexin A1 (ANXA1) expression with epithelial-mesenchymal transition (EMT), angiogenesis, and stromal cells in The Cancer Genome Atlas (TCGA) and GSE57495 cohorts. (A) Scatter plots of ANXA1 expression with the EMT score. (B) Boxplots of EMT-associated gene expression: cadherin1 (CDH1) and $C D H 2$, snail family transcriptional repressor 1 (SNAI1) and SNAI2, and twist-related protein 1 (TWIST1) by ANXA1 low and ALXA1 high pancreatic cancer (PC). (C) Scatter plots of ANXA1 expression with the angiogenesis score. (D) Boxplots of infiltrating fraction of endothelial cells, microvascular endothelial (mvE) cells, and lymphatic endothelial (lyE) cells by ANXA1 low and ANXA1 high PC. (E) Boxplots of angiogenesis-associated genes expression: platelet and endothelial cell adhesion molecule 1 (PECAM1) and sphingosine-1-phosphate receptor 1 (S1PR1) by ANXA1 low and ANXA1 high PC. (F) Boxplots of the infiltrating fraction of fibroblasts and keratinocytes by ANXA1 low and ANXA1 high PC. Median cut-off within each cohort was used to divide them into ANXA1 low and ANXA1 high groups ( $n=88$, respectively, in the TCGA and $n=31$ and 32, respectively, in the GSE57495 cohort). Spearman's rank correlation was used for the correlation analysis. For group comparison, $p$-values were calculated by the Mann-Whitney $U$ test.

\subsection{Annexin A1 (ANXA1) High PC Enriched Multiple Malignant Pathways}

To better understand the functional characteristics of ANXA1 high PC, we performed a pathway analysis using the Gene Set Enrichment Analysis (GSEA) with Hallmark gene sets in the TCGA and GSE57495 cohorts. In both cohorts, ANXA1 high PC consistently enriched multiple malignant pathways such as hypoxia, transforming growth factor (TGF)$\beta$ signaling, TNF- $\alpha$ signaling via nuclear factor-kappa B (NF-kB), and MTORC1, as well as apoptosis, protein secretion, glycolysis, and the androgen response (Figure 2; all gene sets; normalized enrichment score $(\mathrm{NES})>1.5$, false discovery rate $(\mathrm{FDR})<0.25)$. These results suggest that ANXA1 high PC is associated with EMT and other malignant pathways in PC.

2.3. ANXA1 High PC Was Associated with Homologous Recombination Deficiency (HRD), TP53 Mutation, and Other Signaling Pathways but Not with Mutation Load

The mutation of TP53, KRAS, CDKN2A, and SMAD4 plays a key role in the carcinogenesis of PC. As such, it was of interest whether ANXA1 expression related to the mutation load and HRD, as well as the mutation rates and signaling pathways of these genes in the TCGA cohort. We found that ANXA1 high PC was not associated with either silent or non-silent mutation rates, fraction altered, single nucleotide variation (SNV), or Indel neoantigens; however, it was associated with HRD (Figure 3A; $p=0.026$ ). ANXA1 high PC was significantly associated with high mutation rates of the TP53 gene but not with KRAS, CDK2A, or SMAD4 (Figure 3B). However, p53, KRAS, E2F, and TGF- $\beta$ signaling pathways, measured by the GSVA algorithm, were all significantly associated with ANXA1 expression (Figure $3 C$, all $p<0.001$ ). These findings suggest that $A N X A 1$ high PC are associated with HRD; TP53 mutation; and the signaling of TP53, KRAS, CDKN2A, and SMAD4. 


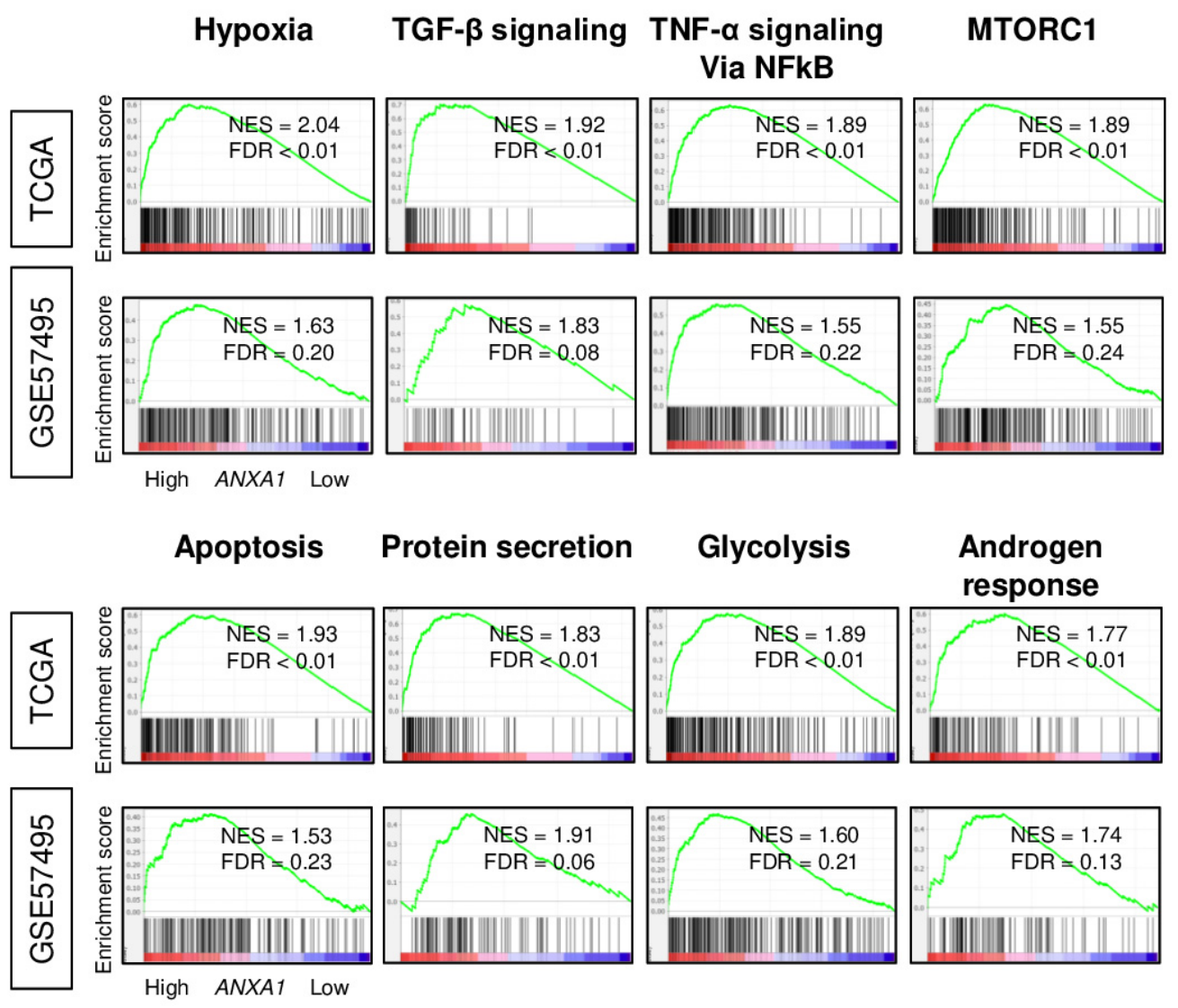

Figure 2. Gene set enrichment analysis of pancreatic cancer with high annexin A1 (ANXA1) expression in the TCGA and GSE57495 cohorts. Enrichment plots of hypoxia, transforming growth factor (TGF)- $\beta$ signaling, tumor necrosis factor (TNF)- $\alpha$ signaling, MTORC1, apoptosis, protein secretion, glycolysis, and the androgen response of hallmark gene sets with NES and FDR. Median cut-off within each cohort was used to divide into ANXA1 low and ANXA1 high groups ( $n=88$, respectively, in the TCGA and $n=31$ and 32, respectively, in the GSE57495 cohort). NES, normalized enrichment score and FDR, false discovery rate.

\subsection{ANXA1 High PC Was Associated with High Infiltration of T-Helper Type 2 (Th2) Cells}

We reported that $A N X A 1$ high breast cancer is associated with a high infiltration of mast cells [15]. Therefore, we wanted to investigate whether similar immune cell infiltration is seen in ANXA1 high PC. The xCell algorithm was used to estimate the fraction of immune cells in the tumor microenvironment of PC in the TCGA and GSE57495 cohorts. We found that ANXA1 high PC was significantly associated with a high infiltration of Th2 cells consistently in both the TCGA and GSE57495 cohorts (Figure 4; $p=0.003$ and $p<0.001$, respectively). Interestingly, $A N X A 1$ high PC was associated with a high infiltration of mast cells in the TCGA; however, it was associated with a low infiltration in the GSE57495 cohort. Furthermore, ANXA1 high PC was associated with a low infiltration of $\mathrm{CD}^{+} \mathrm{T}$ cells and Th1 cells in the GSE57495 cohort but not in the TCGA. These findings suggest that a high expression of ANXA1 is associated with a high infiltration of Th2 cells in PC. 


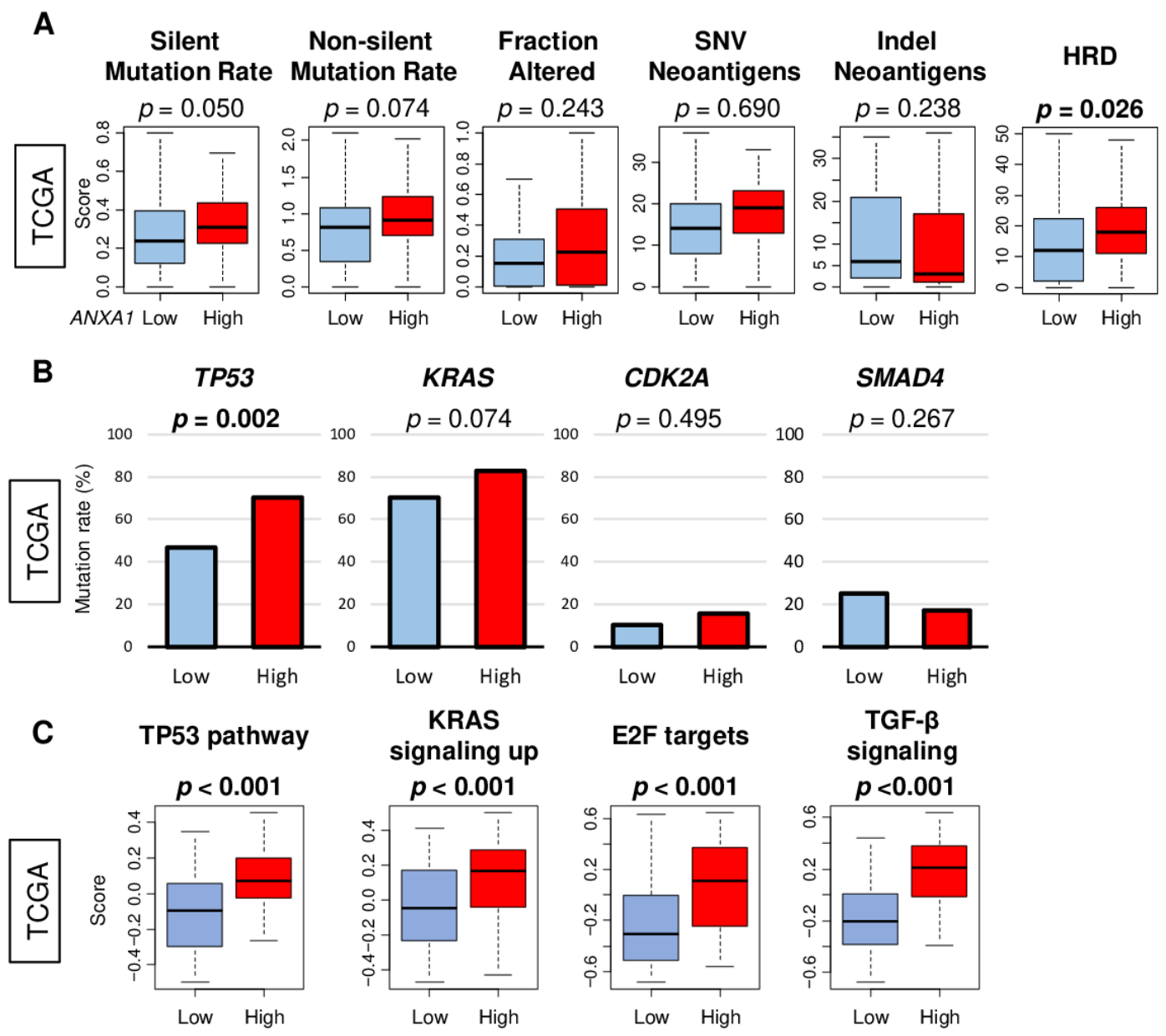

Figure 3. The association of $A N X A 1$ with gene mutation and homologous recombination deficiency (HRD) in the TCGA pancreatic cancer cohort. (A) Bar plots of the mutation rate of KRAS, TP53, CDK2A, and SMAD4 by ANXA1 low and ANXA1 high groups. $p$-values were calculated with Fisher's exact test. (B) Boxplots of the mutation-related scores, including altered fraction, single nucleotide variant (SNV) and indel neoantigens, and silent and non-silent mutations, and (C) HRD scores by ANXA1 low (blue) and ANXA1 high (red) groups. Median cut-off was used to divide into ANXA1 low and ANXA1 high groups ( $n=88$, respectively). $p$-values were calculated by the Mann-Whitney $U$ test.

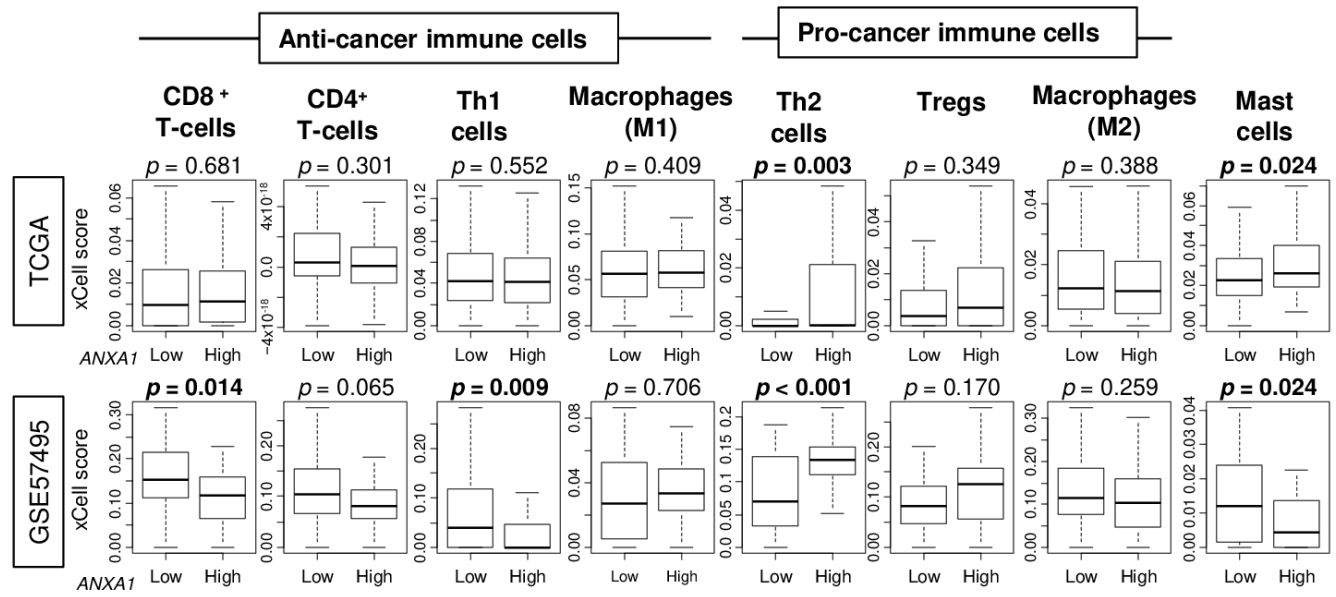

Figure 4. Association of the ANXA1 expression and infiltrating immune cells in pancreatic cancer in the TCGA and GSE57495 cohorts. Boxplots of the infiltrating fraction of CD8 ${ }^{+} \mathrm{T}$ cells, CD4 ${ }^{+} \mathrm{T}$ cells, T-helper types 1 and 2 (Th1 and Th2), regulatory T cells (Tregs), M1 and M2 macrophages, and Mast cells by ANXA1 low and ANXA1 high groups. Median cut-off within each cohort was used to divide into ANXA1 low and $A N X A 1$ high groups $(n=88$, respectively, in the TCGA and $n=31$ and 32, respectively, in the GSE57495 cohort). $p$-values were calculated by the Mann-Whitney $U$ test. 


\subsection{ANXA1 High PC Is Associated with Advanced Histological Grade and with Increased Cell Proliferation}

Given that ANXA1 high PC was associated with multiple malignant pathways, including EMT, which, in turn, was related to metastasis, we expected ANXA1 high PC to be associated with the clinical parameters of aggressiveness in PC. Although ANXA1 expression was not associated with the American Joint Committee on Cancer (AJCC) Stage and lymph node metastasis (N-category) (Figure 5A; $p=0.73$ and 0.582 , respectively), ANXA1 high PC was significantly associated with the advanced histologic grade (Figure 5A; $p<0.001)$. We also investigated the association of the ANXA1 expression with the clinical features (age at diagnosis, race, AJCC T- and M-categories, primary tumor site, and histological diagnosis) in the TCGA cohort. There was no significant difference between ANXA1 low and ANXA1 high PC (Supplemental Table S1). On the other hand, ANXA1 high PC was significantly associated with a high proliferation score and a high expression of MKI67 (Figure 5B; both $p<0.001$ ). Furthermore, ANXA1 expression was significantly correlated with cell proliferation-related pathways: E2F targets, the G2M checkpoint, and Mitotic spindle, which were calculated by the GSVA algorithm (Figure 5C; E2F targets; Spearman's rank correlation $(r)=0.457$ and 0.392 , G2M checkpoint; $r=0.502$ and 0.391 , Mitotic spindle; $r=0.592$ and 0.452 , respectively, all $p<0.01)$. These findings suggest that ANXA1 expression significantly correlates with aggressive cell proliferation in PC.

A

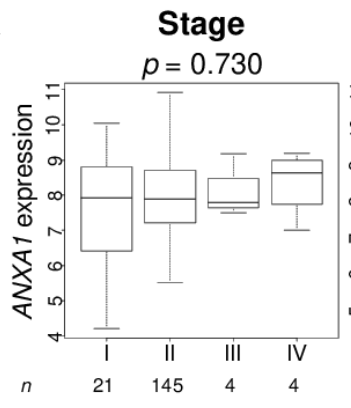

N-category $p=0.582$

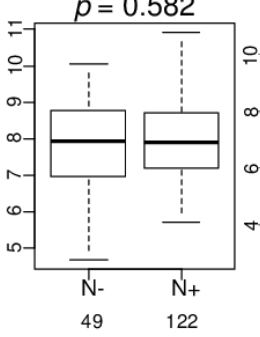

Grade $p<0.001$

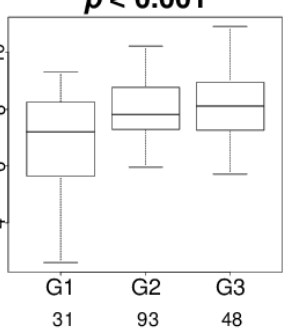

B Proliferation

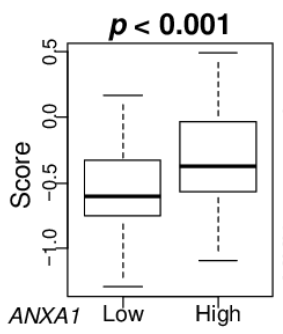

MKI67

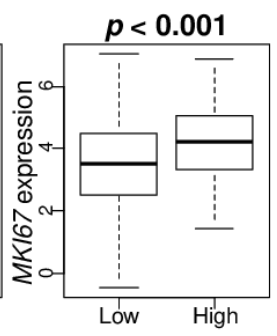

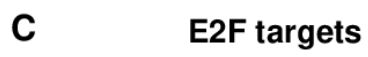

G2M checkpoint

Mitotic spindle

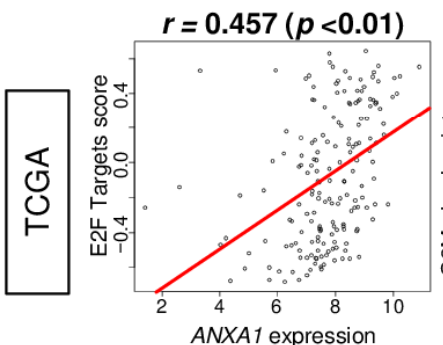

$r=0.502(p<0.01)$ $r=0.592(p<0.01)$
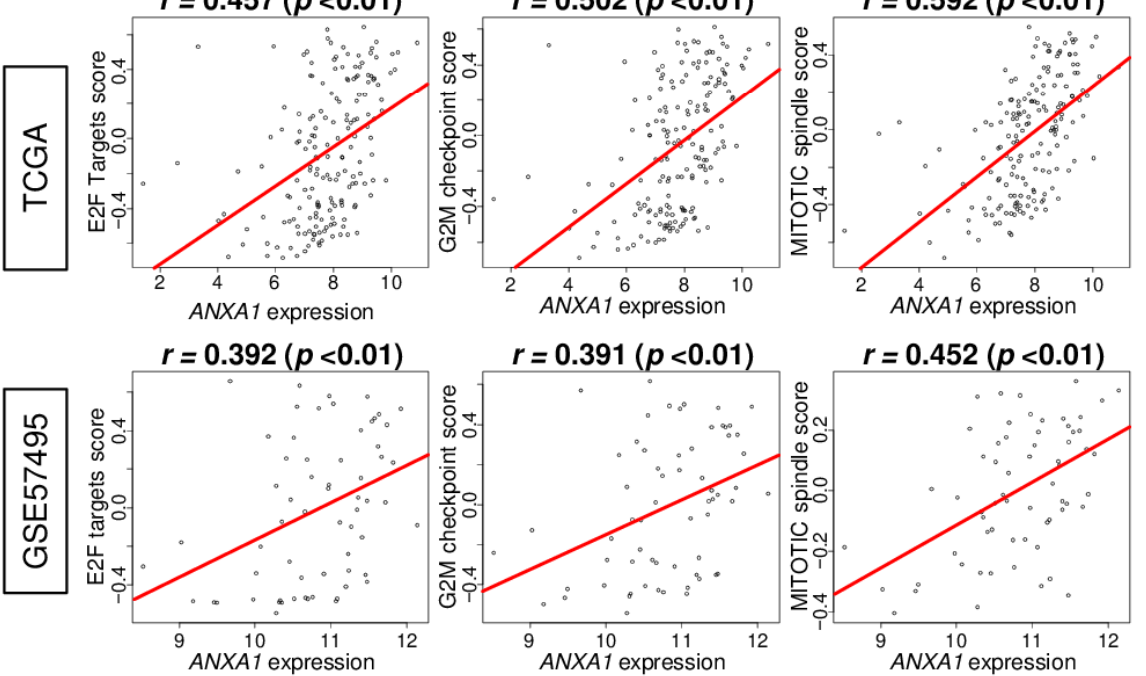

Figure 5. Association of the ANXA1 expression and tumor aggressiveness in PC. Boxplots of (A) the clinical factors: AJCC stage, N-category, and histological grade and (B) proliferation-related factors: proliferation score and MKI67 expression in the TCGA cohort. $p$-values were calculated by the Kruskal-Wallis and Mann-Whitney $U$ tests. (C) Correlation plots between the ANXA1 expression and cell proliferation-related score: E2F targets, G2M checkpoint, and Mitotic spindle score in the TCGA and GSE57495 cohorts. The median cut-off was used to divide them into the ANXA1 low and ANXA1 high groups ( $n=88$, respectively). Spearman's rank correlation was used for the analysis. AJCC: American Joint Committee on Cancer. 


\subsection{ANXA1 High PC Are Significantly Associated with Worse Survival}

Given that ANXA1 expression is significantly correlated with cancer cell proliferation, we predicted that ANXA1 high PC is associated with worse survival. To assess this, we analyzed the disease-free survival (DFS), disease-specific survival (DSS), and progressionfree survival (PFS) in the TCGA cohort, as well as the overall survival (OS) in the TCGA and GSE57495 cohorts. We found that ANXA1 high PC was significantly associated with worse DFS, DSS, and PFS in the TCGA, as well as worse OS in both cohorts (Figure 6A; all $p<0.05$ ). We also analyzed whether the survival risk is the same by histological grade, since the ANXA1 expression was higher in the advanced histological grade. We found that ANXA1 high PC was significantly associated with a worse PFS regardless of grade 1 , grade 2, or grade 3 (Figure 6; $p=0.040$ and 0.018 , respectively). These results indicate that, prognostically, an ANXA1 high expression is significantly associated with worse survival in PC patients regardless of histological grade.

A

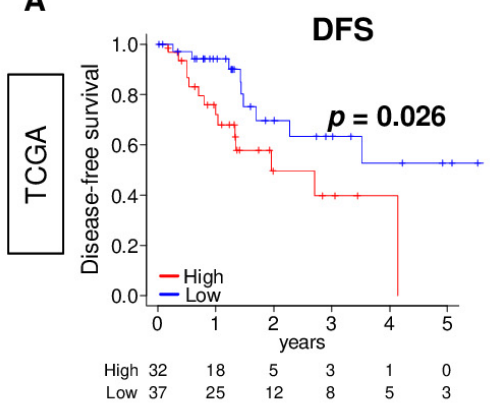

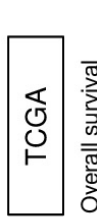

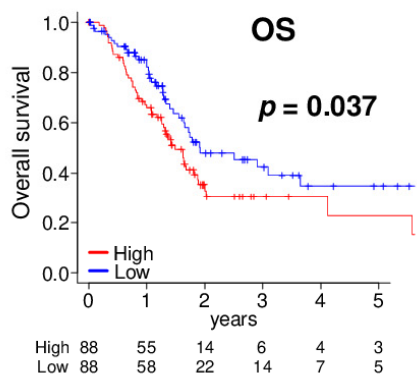

B

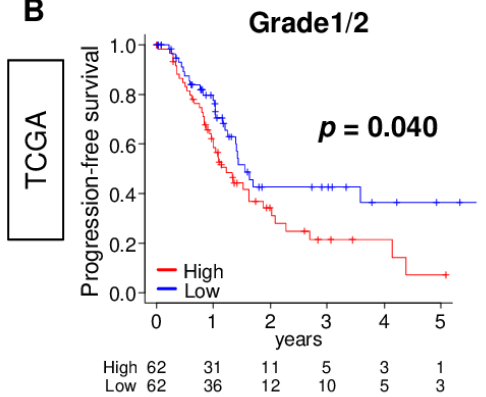

DSS

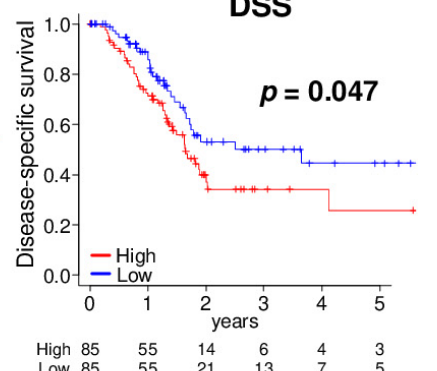

$\begin{array}{lllcll}\text { High } 85 & 55 & 14 & 6 & 4 & 3 \\ \text { Low } 85 & 55 & 21 & 13 & 7 & 5\end{array}$
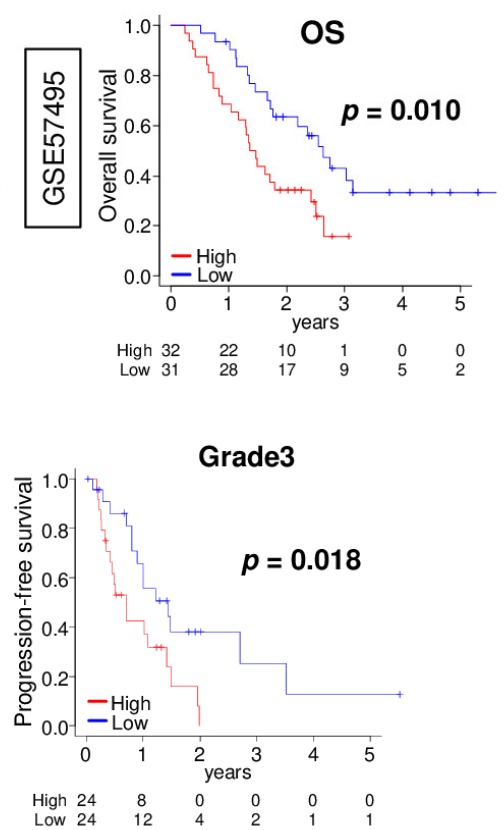

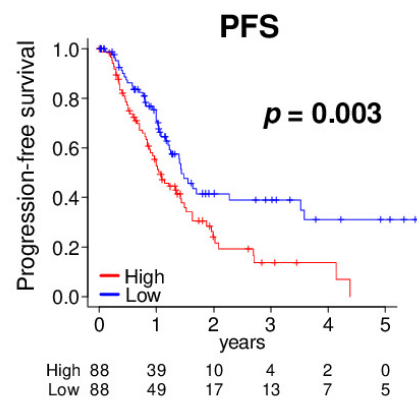

$\begin{array}{llllll}\text { High } 88 & 39 & 10 & 4 & 2 & 0 \\ \text { Low 88 } & 49 & 17 & 13 & 7 & 5\end{array}$

Figure 6. Association of the ANXA1 expression with patient survival in the TCGA and GSE57495 cohorts. (A) Kaplan-Meier curve between low (blue line) and high (red line) with disease-free survival (DFS), disease-specific survival (DSS), progression-free survival (PFS), and overall survival (OS). The median cut-off within each cohort was used to divide them into the ANXA1 low and ANXA1 high groups ( $n=88$, respectively, in the TCGA and $n=31$ and 32, respectively, in the GSE57495 cohort). (B) Kaplan-Meier curve between low and high with PFS in the pathological grades 1/2 ( $n=62$, respectively) and grade 3 groups ( $n=24$, respectively) in the TCGA cohort. $p$-values were calculated by log-rank test. 


\subsection{ANXA1 Expression Correlates with Drug Sensitivity in PC Cells}

Finally, we investigated the association of ANXA1 expression and drug response in PC cells. The list of cells used for analyses is shown in Supplemental Table S3. We found that ANXA1 expression was significantly correlated with the area under the curve (AUC) of gemcitabine and doxorubicin in the primary PC cell lines (Figure 7; Spearman's rank correlate coefficient ${ }^{\circledR}=0.587(p=0.03)$ and $r=0.535(p=0.03)$, respectively) and negatively correlated with the AUC of 5-fluorouracil in the metastatic PC cell lines (Figure 7; $r=-0.553(p=0.01))$. These results implicate that ANXA1 expression may be associated with drug responses in PC that warrant further study.

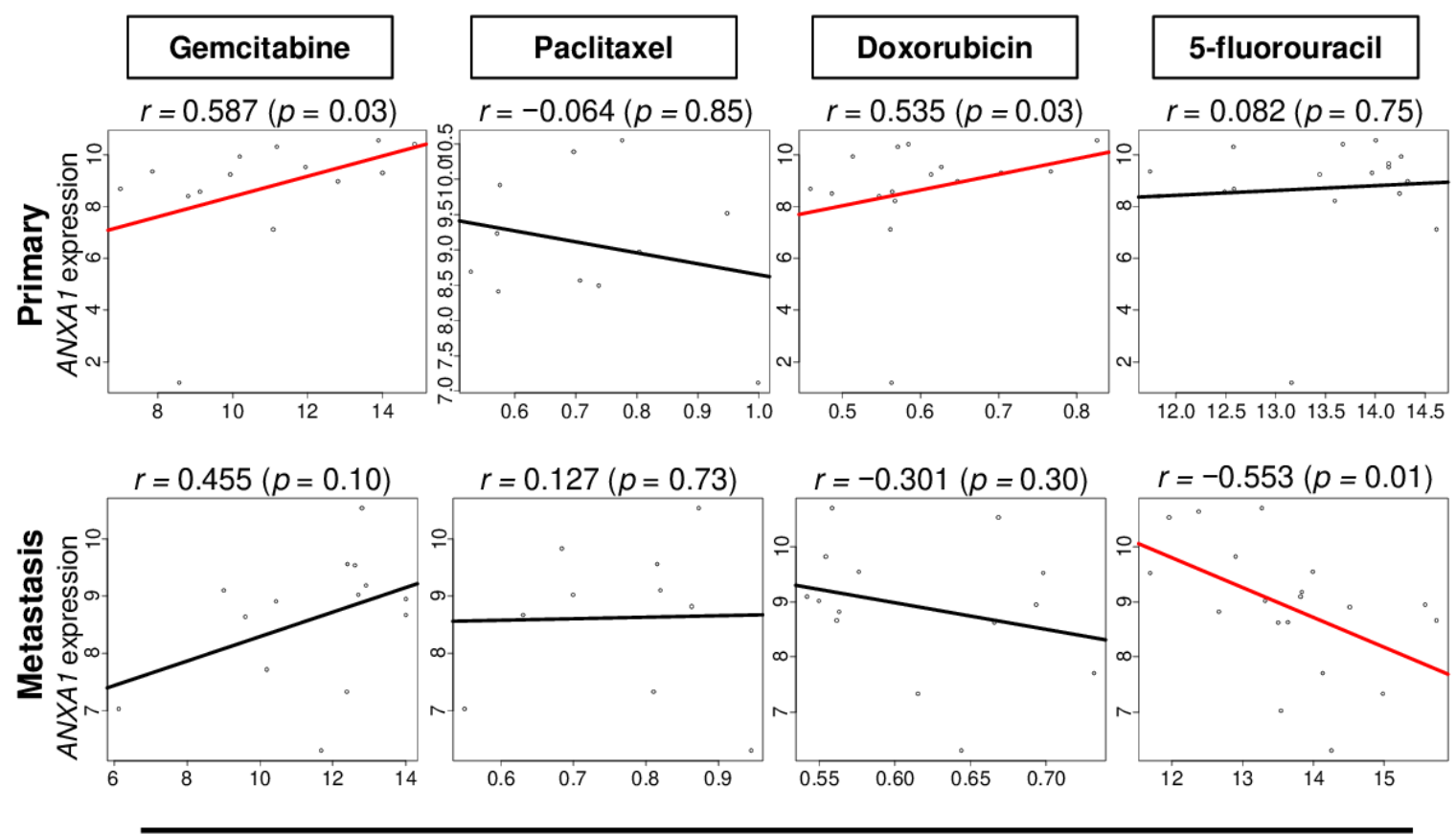

Drug sensitivity AUC

Figure 7. Correlation of the ANXA1 expression with the drug sensitivity of several drugs in primary and metastasis PC cell lines. The correlation plots of the ANXA1 expression with the level of drug sensitivity area under the curve (AUC) of gemcitabine, paclitaxel, doxorubicin, and 5-fluorouracil. Spearman's rank correlation coefficient was used for the analysis.

\section{Discussion}

Recent advances in sequencing technology and genetic analyses allow us to investigate the functions of cancer from the transcriptome of a bulk tumor. Analyses using GSVA scores allow investigators to explore the biologic activity of varying signaling pathways and help identify the mechanisms involved. This approach is well-accepted in the field, evident from numerous citations of the original paper. Our group previously reported that the angiogenesis score was significantly associated with specific gene expressions such as VEGF-related genes, endothelial cell marker genes, and vascular-stability-related genes using this approach [24]. Cell proliferation-related pathways such as E2F targets [23], G2M checkpoint [25], and MYC target [26] scores were significantly associated with the pathological grade in breast cancer. The clinical relevance of angiogenesis [24], inflammation [27], cell proliferation-related pathways [5,23,25,28], KRAS signaling [29], and estrogen response pathways [22] have been published using the same approach. Furthermore, the xCell algorithm allows us to measure several types of cells, including immune cells and stromal cells, in the tumor microenvironment (TME). Our group previously reported the clinical relevance of $\mathrm{CD}^{+} \mathrm{T}$ cells [30], regulatory T cells [31], and dendritic cells (DC) [32], as well as fibroblasts [8], in multiple types of cancer using the xCell algorithm. The link 
between ANXA1 expression and several signaling pathways and several cells in TME was elucidated using these algorithms.

Specifically, in this study, we investigated the clinical relevance of ANXA1 expression in pancreatic cancer (PC). ANXA1 expression correlated with EMT and its related gene expressions but very weakly with angiogenesis and had no relationship with vascular cells or mature blood vessels in PC. ANXA1 high PC was significantly associated with a high fraction of fibroblasts and keratinocytes in the tumor microenvironment (TME). ANXA1 high PC enriched multiple malignant pathways, including hypoxia, TNF- $\alpha$ signaling via NF-kB, and MTORC1, as well as the apoptosis, protein secretion, glycolysis, and androgen response gene sets by GSEA. ANXA1 expression was associated with TP53 mutation alone but was associated with all the KRAS, p53, E2F, and TGF- $\beta$ signaling pathways. ANXA1 high PC was associated with homologous recombination deficiency but not with mutation load.

Additionally, ANXA1 high PC was associated with high infiltration of T-helper type 2 cells in TME. Furthermore, ANXA1 high PC was associated with the advanced histologic grade, cell proliferation, and MKI67 expression. From a prognostic standpoint, ANXA1 high PC was significantly associated with worse patient survival regardless of the grade. Finally, high ANXA1 expression correlated positively with the sensitivity to gemcitabine and doxorubicin and negatively with the sensitivity to 5-fluorouracil in PC cell lines.

Although numerous publications demonstrate that ANXA1 plays multifaceted roles in cancer development and progression, its expression and function appear to be "cancer typespecific" [33]. ANXA1 activation was reported to play a critical role in the EMT pathway in several types of cancer, including PC, consistent with our results. Interestingly, $C D H 1$, an epithelial marker that was shown to decrease with EMT, was elevated in ANXA1 high PC in this current study, which coincides with the previous report that $C D H 1$ correlates with EMT [34]. Since almost all the genes in the EMT pathway gene set significantly correlated with ANXA1 expression, we concluded the association. ANXA1 protein could regulate metastasis by favoring cell migration/invasion intracellularly, as a cytoskeleton remodeling factor, and extracellularly like the formyl peptide receptor (FPR) ligand [11,35]. We found that not only EMT but also other signaling pathways such as protein secretion, glycolysis, and the androgen response were enriched in ANXA1 high PC. Although ANXA1 was initially found to inhibit the inflammatory response, that has not been shown in cancer models [20]. However, our group previously reported that ANXA1 high breast cancer was significantly associated with inflammation and angiogenesis signaling pathways [15]. The fact that the association with inflammation or angiogenesis was not observed in PC indicates that the relationship between ANXA1 expression and inflammation or angiogenesis may differ by the cancer type.

The gold standard to analyze the tumor microenvironment (TME), which plays a crucial role in cancer progression and treatment response, is flow cytometry or immunohistochemistry. Although these approaches are well-established, they are expensive and labor-intensive, particularly when analyzing large patient cohorts. By comparison, bioinformatic approaches can estimate the quantity and function of cells in tens of thousands of samples with less cost and time [4,36,37]. We previously reported the clinical relevance of immune cells, including $\mathrm{CD} 8^{+} \mathrm{T}$ cells [30], regulatory $\mathrm{T}$ cells [31], and dendritic cells (DC) [32], as well as stromal cells such as fibroblasts [8], in the TME using the xCell algorithm, which allows us to estimate the fraction of 64 cells in the TME with the transcriptome of a bulk tumor. Cancer of the breast, colorectal, lung, and kidney with a low expression of ANXA1 is scarcely infiltrated by DC and cytotoxic T lymphocytes, supporting the idea that $A N X A 1$ deficiency facilitates immune escape [38]. The association between ANXA1 and the tumor immune microenvironment in PC has not been fully studied. In this study, ANXA1 high PC was found to be associated with a high infiltration of Th2 cells. Since Th2 cells are known as pro-cancer immune cells [39], high Th2 cell infiltration may explain the association between $A N X A 1$ high and poor prognosis in PC patients. We previously reported that $A N X A 1$ high breast cancer was significantly associated with high infiltration 
of mast cells [15]. Since the functions of $A N X A 1$ and mast cells vary in different cancer types, the association between ANXA1 expression and mast cells may differ between breast cancer and PC. Several studies, including one using ANXA1 knockout mice, showed that stroma-derived $A N X A 1$ expression promotes tumor growth, angiogenesis, and metastasis [16,17]. The relationship between $A N X A 1$ and fibroblasts that we showed is in line with that report. It is possible that $A N X A 1$ generated by fibroblasts contributes to its expression in bulk tumors and is one of the reasons why $A N X A 1$ expression was highly associated with poor prognosis.

Drug resistance is one of the major obstacles that contribute to PC mortality in clinics. Whether it is de novo or acquired, drug resistance involves numerous genetic and epigenetic alterations in PC. Numerous studies have attempted to identify the mechanisms and molecular markers involved in either de novo or acquired drug resistance processes $[40,41]$. Zhang et al. reported that the overexpression of $A N X A 1$ induced by low-concentration arsenic trioxide (ATO), an antitumor agent, makes cancer cells more resistant to the agent via activated ERK MAPKs [42]. Belvedere et al. reported that ANXA1 expression maintains an overall aggressive phenotype and chemotherapy resistance using ANXA1 knockout MIA PaCa-2 cells [11]. We showed results similar to this report with human patient data. We also showed the association of $A N X A 1$ expression with proliferation-related factors, such as MKI67 expression, as well as gene sets of the E2F targets, mitotic spindle, and G2M checkpoints. This finding is in the setting of previous reports that high G2M checkpoint activity is significantly associated with a worse prognosis in PC [5].

Interestingly, we found that $A N X A 1$ is positively correlated to the apoptosis pathway, where evading apoptosis is one of the original hallmarks of cancer [43]. We believe this is because we analyzed the involvement of ANXA1 in cancer progression-the worsening of existing cancer. This is different from the hallmarks of cancer, which are mechanisms in carcinogenesis, the development of cancer from normal cells. Further, our findings suggest that ANXA1 high PC is associated with overwhelming cell proliferation that overrides elevated apoptosis, which may be, in part, due to activation of the p53 pathway. In the current study, we demonstrated that ANXA1 expression is associated with poor survival, which coincides with Shang et al., who analyzed 39 pancreatic ductal adenocarcinoma patients [44], where we analyzed 239 PC patients. These results suggest that future studies investigating the relationship between $A N X A 1$ expression and drug response using large clinical cohorts are warranted.

We successfully demonstrated the clinical relevance of ANXA1 in PC; however, this study still had certain limitations. This study was prone to selection bias, given it was a retrospective design using previously published cohorts. Additionally, we were able to use these cohorts to evaluate the gene expression at a single time point, at the time of surgical removal of $\mathrm{PC}$, but not evaluate the change in gene expression and signaling pathways in these tumors over time. The relationship between ANXA1 expression and the drug response was assessed using data from the cell line encyclopedia, since we did not have access to any PC patient cohorts with comprehensive transcriptomes that were associated with clinical drug response data. A prospective study will be required to prove the utility of $A N X A 1$ expression as a prognostic and predictive biomarker. In particular, ANXA1 expression is expected to be a useful tool to identify which chemotherapy may respond to which patient and when by obtaining tumor samples longitudinally, including metastatic tumors.

\section{Materials and Methods}

\subsection{Clinical and Transcriptomic Data of TCGA and GEO Cohorts in Pancreatic Cancer Patients}

Transcriptomic data and mutation data of The Cancer Genome Atlas of pancreatic adenocarcinoma (TCGA-PAAD; $n=176$ ) were obtained through the cBio Cancer Genomic Portal [45]. Survival data of pancreatic cancer patients were obtained from the Pan-Cancer Clinical Data Resource [46]. Clinical and transcriptomic data of the GSE57495 cohort 
studied by Chen et al. ( $n=63)$ [47] were obtained through the Gene Expression Omnibus (GEO) repository. $\log _{2}$-transformed gene expression data were used for all analyses.

\subsection{Drug Sensitivity and Transcriptomic Data of Pancreatic Cancer Cell Lines}

Thirty-five pancreatic cancer cell lines with both comprehensive gene expression and drug response (area under the curve (AUC)) data from the cancer cell line encyclopedia (CCLE) [48] through the Depmap portal (https://depmap.org/portal/, accessed on 1 February 2021) were used to assess the correlation between ANXA1 expression and the drug response. The AUCs were adjusted for the range of tested drug concentrations that allowed the integration of heterogeneous drug sensitivity data from the CCLE, the Genomics of Drug sensitivity in cancer (GDSC), and the Cancer Therapeutics Response Portal (CTRP) [49]. The list of cell lines is shown in Table S3.

\subsection{Scores}

We used several scores in this study. Homologous recombinant deficiency and mutation-related, including altered fraction, single nucleotide variant (SNV) and indel neoantigens, and silent and non-silent mutations, were calculated by Thorsson et al. in the TCGA cohort [50]. Gene set scores, including angiogenesis, E2F, G2M, and EMT, were calculated by the gene set variation analysis (GSVA) with hallmark gene sets of the Molecular Signatures Database (MSigDB) collection, as we previously reported $[5,23,24,51]$. We used the $x$ Cell score as the infiltrating fraction of the immune and stromal cells in the tumor microenvironment, which was calculated by the xCell algorithm [4], as we previously reported [52-55].

\subsection{Statistical Analysis}

$\mathrm{R}$ software (v 4.0.1, R project for Statistical Computing) and Microsoft Excel (v 16 for Windows, Redmond, WA, USA) were to analyze and generate the graphs in the study. We divided them into ANXA1 low and ANXA1 high groups by the median cut-off within each cohort. $p$-values were calculated by Fisher's exact test. The Kruskal-Wallis and Mann-Whitney $U$ test were used for group comparisons, as described in their respective figure legends. The Log-rank test was used for the survival analysis. $p$-values $<0.05$ were used to determine the statistical significance.

\section{Conclusions}

Annexin A1 (ANXA1) expression is associated with EMT; multiple malignant pathways; and the infiltration of fibroblasts, keratinocytes, and T-helper type 2 cells in the tumor microenvironment. Furthermore, ANXA1 high PC expression is associated with cell proliferation and worse patient survival and drug response.

Supplementary Materials: The following are available online at https:/ /www.mdpi.com/2073-440 9/10/3/653/s1: Table S1: Correlation between ANXA1 expression and EMT-associated genes in the hallmark EMT gene sets in the TCGA cohort. Table S2: Clinical and pathological features between ANXA1 low and ANXA1 high pancreatic cancer in the TCGA cohort. Table S3: Pancreatic cancer cell lines. Figure S1: Histogram of ANXA1 expression in the TCGA and GSE57495 cohorts. Figure S2: Association of ANXA1 with epithelial-mesenchymal transition (EMT)-associated genes in the TCGA and GSE57495 cohorts.

Author Contributions: Conceptualization, I.E., K.T., M.O., and R.M.; methodology, K.T., M.O., and Y.T.; formal analysis, M.O. and L.Y.; writing—original draft preparation, M.O.; writing—review and editing, K.T., L.Y., and S.M.; supervision, K.T.; and project administration, K.T. All authors have read and agreed to the published version of the manuscript. 
Funding: This work was supported by US National Institutes of Health/National Cancer Institute grant R01CA160688, R01CA250412, R37CA248018, US Department of Defense BCRP grant W81XWH19-1-0674, as well as the Edward K. Duch Foundation and Paul \& Helen Ellis Charitable Trust to K.T., and US National Cancer Institute cancer center support grant P30-CA016056 to Roswell Park Comprehensive Cancer Center.

Institutional Review Board Statement: Not applicable.

Informed Consent Statement: Not applicable.

Data Availability Statement: All data were from previous studies.

Conflicts of Interest: The authors declare no conflict of interest.

\section{References}

1. Rawla, P.; Sunkara, T.; Gaduputi, V. Epidemiology of Pancreatic Cancer: Global Trends, Etiology and Risk Factors. World J. Oncol. 2019, 10, 10-27. [CrossRef]

2. Masetti, M.; Acquaviva, G.; Visani, M.; Tallini, G.; Fornelli, A.; Ragazzi, M.; Vasuri, F.; Grifoni, D.; Di Giacomo, S.; Fiorino, S.; et al. Long-term survivors of pancreatic adenocarcinoma show low rates of genetic alterations in KRAS, TP53 and SMAD4. Cancer Biomark. Sect. A Dis. Markers 2018, 21, 323-334. [CrossRef]

3. Hänzelmann, S.; Castelo, R.; Guinney, J. GSVA: Gene set variation analysis for microarray and RNA-seq data. BMC Bioinform. 2013, 14, 7. [CrossRef]

4. Aran, D.; Hu, Z.; Butte, A.J. xCell: Digitally portraying the tissue cellular heterogeneity landscape. Genome Biol. 2017, 18, 220. [CrossRef]

5. Oshi, M.; Newman, S.; Tokumaru, Y.; Yan, L.; Matsuyama, R.; Endo, I.; Katz, M.H.G.; Takabe, K. High G2M Pathway Score Pancreatic Cancer is Associated with Worse Survival, Particularly after Margin-Positive (R1 or R2) Resection. Cancers 2020, 12, 2871. [CrossRef]

6. Takahashi, H.; Katsuta, E.; Yan, L.; Tokumaru, Y.; Katz, M.H.G.; Takabe, K. Transcriptomic Profile of Lymphovascular Invasion, a Known Risk Factor of Pancreatic Ductal Adenocarcinoma Metastasis. Cancers 2020, 12, 2033. [CrossRef]

7. Katsuta, E.; Qi, Q.; Peng, X.; Hochwald, S.N.; Yan, L.; Takabe, K. Pancreatic adenocarcinomas with mature blood vessels have better overall survival. Sci. Rep. 2019, 9, 1310. [CrossRef]

8. Katsuta, E.; Rashid, O.M.; Takabe, K. Fibroblasts as a Biological Marker for Curative Resection in Pancreatic Ductal Adenocarcinoma. Int. J. Mol. Sci. 2020, 21, 3890. [CrossRef]

9. Bizzarro, V.; Petrella, A.; Parente, L. Annexin A1: Novel roles in skeletal muscle biology. J. Cell. Physiol. 2012, 227, 3007-3015. [CrossRef]

10. Gerke, V.; Moss, S.E. Annexins: From structure to function. Physiol. Rev. 2002, 82, 331-371. [CrossRef]

11. Belvedere, R.; Bizzarro, V.; Forte, G.; Dal Piaz, F.; Parente, L.; Petrella, A. Annexin A1 contributes to pancreatic cancer cell phenotype, behaviour and metastatic potential independently of Formyl Peptide Receptor pathway. Sci. Rep. 2016, 6, 29660. [CrossRef] [PubMed]

12. Bai, X.F.; Ni, X.G.; Zhao, P.; Liu, S.M.; Wang, H.X.; Guo, B.; Zhou, L.P.; Liu, F.; Zhang, J.S.; Wang, K.; et al. Overexpression of annexin 1 in pancreatic cancer and its clinical significance. World J. Gastroenterol. 2004, 10, 1466-1470. [CrossRef] [PubMed]

13. Perretti, M.; D'Acquisto, F. Annexin A1 and glucocorticoids as effectors of the resolution of inflammation. Nat. Rev. Immunol. 2009, 9, 62-70. [CrossRef]

14. De Graauw, M.; van Miltenburg, M.H.; Schmidt, M.K.; Pont, C.; Lalai, R.; Kartopawiro, J.; Pardali, E.; Le Dévédec, S.E.; Smit, V.T.; van der Wal, A.; et al. Annexin A1 regulates TGF-beta signaling and promotes metastasis formation of basal-like breast cancer cells. Proc. Natl. Acad. Sci. USA 2010, 107, 6340-6345. [CrossRef]

15. Okano, M.; Oshi, M.; Butash, A.L.; Katsuta, E.; Tachibana, K.; Saito, K.; Okayama, H.; Peng, X.; Yan, L.; Kono, K.; et al. TripleNegative Breast Cancer with High Levels of Annexin A1 Expression Is Associated with Mast Cell Infiltration, Inflammation, and Angiogenesis. Int. J. Mol. Sci. 2019, 20, 4197. [CrossRef]

16. Geary, L.A.; Nash, K.A.; Adisetiyo, H.; Liang, M.; Liao, C.P.; Jeong, J.H.; Zandi, E.; Roy-Burman, P. CAF-secreted annexin A1 induces prostate cancer cells to gain stem cell-like features. Mol. Cancer Res. MCR 2014, 12, 607-621. [CrossRef]

17. Yi, M.; Schnitzer, J.E. Impaired tumor growth, metastasis, angiogenesis and wound healing in annexin A1-null mice. Proc. Natl. Acad. Sci. USA 2009, 106, 17886-17891. [CrossRef]

18. Novizio, N.; Belvedere, R.; Pessolano, E.; Tosco, A.; Porta, A.; Perretti, M.; Campiglia, P.; Filippelli, A.; Petrella, A. Annexin A1 Released in Extracellular Vesicles by Pancreatic Cancer Cells Activates Components of the Tumor Microenvironment, through Interaction with the Formyl-Peptide Receptors. Cells 2020, 9, 2719. [CrossRef]

19. Shin, J.; Song, I.S.; Pak, J.H.; Jang, S.W. Upregulation of annexin A1 expression by butyrate in human melanoma cells induces invasion by inhibiting E-cadherin expression. Tumour Biol. J. Int. Soc. Oncodev. Biol. Med. 2016, 37, 14577-14584. [CrossRef]

20. Pessolano, E.; Belvedere, R.; Bizzarro, V.; Franco, P.; Marco, I.; Porta, A.; Tosco, A.; Parente, L.; Perretti, M.; Petrella, A. Annexin A1 May Induce Pancreatic Cancer Progression as a Key Player of Extracellular Vesicles Effects as Evidenced in the In Vitro MIA PaCa-2 Model System. Int. J. Mol. Sci. 2018, 19, 3878. [CrossRef] 
21. Tang, L.; Chen, Y.; Chen, H.; Jiang, P.; Yan, L.; Mo, D.; Tang, X.; Yan, F. DCST1-AS1 Promotes TGF- $\beta$-Induced EpithelialMesenchymal Transition and Enhances Chemoresistance in Triple-Negative Breast Cancer Cells via ANXA1. Front. Oncol. 2020, 10, 280. [CrossRef]

22. Oshi, M.; Tokumaru, Y.; Angarita, F.A.; Yan, L.; Matsuyama, R.; Endo, I.; Takabe, K. Degree of Early Estrogen Response Predict Survival after Endocrine Therapy in Primary and Metastatic ER-Positive Breast Cancer. Cancers 2020, 12, 3557. [CrossRef]

23. Oshi, M.; Takahashi, H.; Tokumaru, Y.; Yan, L.; Rashid, O.M.; Nagahashi, M.; Matsuyama, R.; Endo, I.; Takabe, K. The E2F Pathway Score as a Predictive Biomarker of Response to Neoadjuvant Therapy in ER+/HER2- Breast Cancer. Cells 2020, $9,1643$. [CrossRef]

24. Oshi, M.; Newman, S.; Tokumaru, Y.; Yan, L.; Matsuyama, R.; Endo, I.; Nagahashi, M.; Takabe, K. Intra-Tumoral Angiogenesis Is Associated with Inflammation, Immune Reaction and Metastatic Recurrence in Breast Cancer. Int. J. Mol. Sci. 2020, 21, 6708. [CrossRef]

25. Oshi, M.; Takahashi, H.; Tokumaru, Y.; Yan, L.; Rashid, O.M.; Matsuyama, R.; Endo, I.; Takabe, K. G2M Cell Cycle Pathway Score as a Prognostic Biomarker of Metastasis in Estrogen Receptor (ER)-Positive Breast Cancer. Int. J. Mol. Sci. 2020, 21, 2921. [CrossRef] [PubMed]

26. Schulze, A.; Oshi, M.; Endo, I.; Takabe, K. MYC Targets Scores Are Associated with Cancer Aggressiveness and Poor Survival in ER-Positive Primary and Metastatic Breast Cancer. Int. J. Mol. Sci. 2020, 21, 8127. [CrossRef] [PubMed]

27. Oshi, M.; Newman, S.; Tokumaru, Y.; Yan, L.; Matsuyama, R.; Endo, I.; Takabe, K. Inflammation Is Associated with Worse Outcome in the Whole Cohort but with Better Outcome in Triple-Negative Subtype of Breast Cancer Patients. J. Immunol. Res. 2020, 2020, 5618786. [CrossRef]

28. Oshi, M.; Tokumaru, Y.; Asaoka, M.; Yan, L.; Satyananda, V.; Matsuyama, R.; Matsuhashi, N.; Futamura, M.; Ishikawa, T.; Yoshida, K.; et al. M1 Macrophage and M1/M2 ratio defined by transcriptomic signatures resemble only part of their conventional clinical characteristics in breast cancer. Sci. Rep. 2020, 10, 16554. [CrossRef] [PubMed]

29. Tokumaru, Y.; Oshi, M.; Katsuta, E.; Yan, L.; Satyananda, V.; Matsuhashi, N.; Futamura, M.; Akao, Y.; Yoshida, K.; Takabe, K. KRAS signaling enriched triple negative breast cancer is associated with favorable tumor immune microenvironment and better survival. Am. J. Cancer Res. 2020, 10, 897-907.

30. Oshi, M.; Asaoka, M.; Tokumaru, Y.; Yan, L.; Matsuyama, R.; Ishikawa, T.; Endo, I.; Takabe, K. CD8 T Cell Score as a Prognostic Biomarker for Triple Negative Breast Cancer. Int. J. Mol. Sci. 2020, 21, 6968. [CrossRef]

31. Oshi, M.; Asaoka, M.; Tokumaru, Y.; Angarita, F.A.; Yan, L.; Matsuyama, R.; Zsiros, E.; Ishikawa, T.; Endo, I.; Takabe, K. Abundance of Regulatory T Cell (Treg) as a Predictive Biomarker for Neoadjuvant Chemotherapy in Triple-Negative Breast Cancer. Cancers 2020, 12, 3038. [CrossRef]

32. Oshi, M.; Newman, S.; Tokumaru, Y.; Yan, L.; Matsuyama, R.; Kalinski, P.; Endo, I.; Takabe, K. Plasmacytoid Dendritic Cell (pDC) Infiltration Correlate with Tumor Infiltrating Lymphocytes, Cancer Immunity, and Better Survival in Triple Negative Breast Cancer (TNBC) More Strongly than Conventional Dendritic Cell (cDC). Cancers 2020, 12, 3342. [CrossRef] [PubMed]

33. Gao, Y.; Chen, Y.; Xu, D.; Wang, J.; Yu, G. Differential expression of ANXA1 in benign human gastrointestinal tissues and cancers. BMC Cancer 2014, 14, 520. [CrossRef] [PubMed]

34. Jung, A.R.; Jung, C.H.; Noh, J.K.; Lee, Y.C.; Eun, Y.G. Epithelial-mesenchymal transition gene signature is associated with prognosis and tumor microenvironment in head and neck squamous cell carcinoma. Sci. Rep. 2020, 10, 3652. [CrossRef]

35. Belvedere, R.; Bizzarro, V.; Popolo, A.; Dal Piaz, F.; Vasaturo, M.; Picardi, P.; Parente, L.; Petrella, A. Role of intracellular and extracellular annexin A1 in migration and invasion of human pancreatic carcinoma cells. BMC Cancer 2014, 14, 961. [CrossRef] [PubMed]

36. Li, T.; Fan, J.; Wang, B.; Traugh, N.; Chen, Q.; Liu, J.S.; Li, B.; Liu, X.S. TIMER: A Web Server for Comprehensive Analysis of Tumor-Infiltrating Immune Cells. Cancer Res. 2017, 77, e108-e110. [CrossRef]

37. Newman, A.M.; Liu, C.L.; Green, M.R.; Gentles, A.J.; Feng, W.; Xu, Y.; Hoang, C.D.; Diehn, M.; Alizadeh, A.A. Robust enumeration of cell subsets from tissue expression profiles. Nat. Methods 2015, 12, 453-457. [CrossRef]

38. Baracco, E.E.; Stoll, G.; Van Endert, P.; Zitvogel, L.; Vacchelli, E.; Kroemer, G. Contribution of annexin A1 to anticancer immunosurveillance. Oncoimmunology 2019, 8, e1647760. [CrossRef]

39. Le, L.; Tokumaru, Y.; Oshi, M.; Asaoka, M.; Yan, L.; Endo, I.; Ishikawa, T.; Futamura, M.; Yoshida, K.; Takabe, K. Th2 cell infiltrations predict neoadjuvant chemotherapy response of estrogen receptor-positive breast cancer. Gland Surg. 2021, 10, 154-165. [CrossRef]

40. Liu, Q.H.; Yong, H.M.; Zhuang, Q.X.; Zhang, X.P.; Hou, P.F.; Chen, Y.S.; Zhu, M.H.; Bai, J. Reduced expression of annexin A1 promotes gemcitabine and 5-fluorouracil drug resistance of human pancreatic cancer. Investig. New Drugs 2020, 38, 350-359. [CrossRef]

41. Onozawa, H.; Saito, M.; Saito, K.; Kanke, Y.; Watanabe, Y.; Hayase, S.; Sakamoto, W.; Ishigame, T.; Momma, T.; Ohki, S.; et al. Annexin A1 is involved in resistance to 5-FU in colon cancer cells. Oncol. Rep. 2017, 37, 235-240. [CrossRef]

42. Zhang, X.; Li, X.; Li, X.; Zheng, L.; Lei, L. ANXA1 silencing increases the sensitivity of cancer cells to low-concentration arsenic trioxide treatment by inhibiting ERK MAPK activation. Tumori 2015, 101, 360-367. [CrossRef]

43. Hanahan, D.; Weinberg, R.A. The hallmarks of cancer. Cell 2000, 100, 57-70. [CrossRef]

44. Shang, M.; Zhang, L.; Chen, X.; Zheng, S. Identification of hub genes and regulators associated with pancreatic ductal adenocarcinoma based on integrated gene expression profile analysis. Discov. Med. 2019, 28, 159-172. 
45. Gao, J.; Aksoy, B.A.; Dogrusoz, U.; Dresdner, G.; Gross, B.; Sumer, S.O.; Sun, Y.; Jacobsen, A.; Sinha, R.; Larsson, E.; et al. Integrative analysis of complex cancer genomics and clinical profiles using the cBioPortal. Sci. Signal. 2013, 6, pl1. [CrossRef]

46. Liu, J.; Lichtenberg, T.; Hoadley, K.A.; Poisson, L.M.; Lazar, A.J.; Cherniack, A.D.; Kovatich, A.J.; Benz, C.C.; Levine, D.A.; Lee, A.V.; et al. An Integrated TCGA Pan-Cancer Clinical Data Resource to Drive High-Quality Survival Outcome Analytics. Cell 2018, 173, 400-416. [CrossRef]

47. Chen, D.T.; Davis-Yadley, A.H.; Huang, P.Y.; Husain, K.; Centeno, B.A.; Permuth-Wey, J.; Pimiento, J.M.; Malafa, M. Prognostic Fifteen-Gene Signature for Early Stage Pancreatic Ductal Adenocarcinoma. PLoS ONE 2015, 10, e0133562. [CrossRef]

48. Barretina, J.; Caponigro, G.; Stransky, N.; Venkatesan, K.; Margolin, A.A.; Kim, S.; Wilson, C.J.; Lehár, J.; Kryukov, G.V.; Sonkin, D.; et al. The Cancer Cell Line Encyclopedia enables predictive modelling of anticancer drug sensitivity. Nature 2012, 483, 603-607. [CrossRef]

49. Pozdeyev, N.; Yoo, M.; Mackie, R.; Schweppe, R.E.; Tan, A.C.; Haugen, B.R. Integrating heterogeneous drug sensitivity data from cancer pharmacogenomic studies. Oncotarget 2016, 7, 51619-51625. [CrossRef]

50. Thorsson, V.; Gibbs, D.L.; Brown, S.D.; Wolf, D.; Bortone, D.S.; Ou Yang, T.H.; Porta-Pardo, E.; Gao, G.F.; Plaisier, C.L.; Eddy, J.A.; et al. The Immune Landscape of Cancer. Immunity 2019, 51, 411-412. [CrossRef]

51. Oshi, M.; Kim, T.H.; Tokumaru, Y.; Yan, L.; Matsuyama, R.; Endo, I.; Cherkassky, L.; Takabe, K. Enhanced DNA Repair Pathway is Associated with Cell Proliferation and Worse Survival in Hepatocellular Carcinoma (HCC). Cancers 2021, 13, 323. [CrossRef]

52. Oshi, M.; Tokumaru, Y.; Patel, A.; Yan, L.; Matsuyama, R.; Endo, I.; Katz, M.H.G.; Takabe, K. A Novel Four-Gene Score to Predict Pathologically Complete (R0) Resection and Survival in Pancreatic Cancer. Cancers 2020, 12, 3635. [CrossRef]

53. Oshi, M.; Newman, S.; Murthy, V.; Tokumaru, Y.; Yan, L.; Matsuyama, R.; Endo, I.; Takabe, K. ITPKC as a Prognostic and Predictive Biomarker of Neoadjuvant Chemotherapy for Triple Negative Breast Cancer. Cancers 2020, 12, 2758. [CrossRef]

54. Oshi, M.; Katsuta, E.; Yan, L.; Ebos, J.M.L.; Rashid, O.M.; Matsuyama, R.; Endo, I.; Takabe, K. A Novel 4-Gene Score to Predict Survival, Distant Metastasis and Response to Neoadjuvant Therapy in Breast Cancer. Cancers 2020, 12, 1148. [CrossRef] [PubMed]

55. Oshi, M.; Angarita, F.A.; Tokumaru, Y.; Yan, L.; Matsuyama, R.; Endo, I.; Takabe, K. High Expression of NRF2 Is Associated with Increased Tumor-Infiltrating Lymphocytes and Cancer Immunity in ER-Positive/HER2-Negative Breast Cancer. Cancers 2020, 12, 3856. [CrossRef] [PubMed] 